\title{
Effect of crystallographic features on tableting behaviour of pharmaceutical actives
}

\author{
Arvind Bansal $^{1}$, Kailas S. Khomane ${ }^{1}$ \\ ${ }^{1}$ Department Of Pharmaceutics, National Institute Of Pharmaceutical Education And, Mohali, India \\ E-mail: bansalarvind@yahoo.com
}

Quality by Design (QbD) initiative of US FDA/ICH requires scientific understanding of critical material attributes and process parameters, and the relationship among them. Knowledge of the mechanical properties of the drugs and excipients plays a crucial role in tablet formulation development. The identification of tableting related problems and establishing their relation with compaction behaviour can help in better parametric control of the process. Plurality of parameters like crystal packing features, and particle properties influence the compaction. Polymorphism offers a unique opportunity to study crystal structure property relationships, by virtue of the constancy of chemical composition that eliminates the contribution of different molecular structures. This allows us to examine influence of molecular level properties on mechanical behaviour of pharmaceutical materials.

In the present work, structure-property relationship between crystal structure and mechanical behaviour was investigated using polymorphic pairs of ranitidine hydrochloride (RAN) form I and II, clopidogrel bisulphate (CLP) form I and II, indomethacin (IMC) form $\mathrm{a}$ and $\mathrm{Y}$, clarithromycin (CLM) Form I and II and febuxostat (FXT) Form Q and Form H1. The present work enabled us to generalize the compaction behaviour of pharmaceutical polymorphs. The polymorph having higher true density (closed crystal structure) resisted deformation under the compaction pressure and showed higher yield stress, yield strength and poor compressibility. However, it offered greater tensile strength by virtue of its higher interparticulate bonding strength. On the other hand, polymorph having lower true density (relatively open crystal structure) easily underwent the deformation and exhibited the lower yield stress, yield strength and greater compressibility. However, polymorphic systems having active slip plane system in its higher density form, do not follow this general trend. Influence of other molecular descriptors like heat of fusion, slip plane systems, weak hydrogen bonding, mechanical hardness and elastic modulus (nanoindentation) towards compaction behaviour of pharmaceutical polymorphs has also been revealed.

The present study also established a novel relationship between crystal packing density and bonding strength of compacted pharmaceuticals. Interparticulate bonding strength of the studied materials follows the order of true density rather than heat of fusion or elastic modulus. It is anticipated that higher true density implies higher number of intermolecular contacts per unit bonding area that offers greater bonding strength to the material undergoing compaction. The greater interparticulate bonding resists crack propagation under applied stress, and hence, offers greater tensile strength at a given porosity. The relationship was validated using ten different active pharmaceutical materials. A linear correlation was observed between tensile strength at zero porosity and true density. Active pharmaceutical ingredients that showed tableting problems like capping and lamination or poor tabletability (e.g. paracetamol, clarithromycin or ibuprofen) were found to possess lower true density values and poorer bonding strength.

[1] Khomane, K. S. et al, (2012). J. Pharm. Sci. 101 (7):2408-16

[2] Khomane, K. S. et al, (2013) Mol. Pharm. 10 (2): 631-39

[3] Khomane, K. S. et al, (2013). J. Pharm. Sci. 102 (12): 4242-45

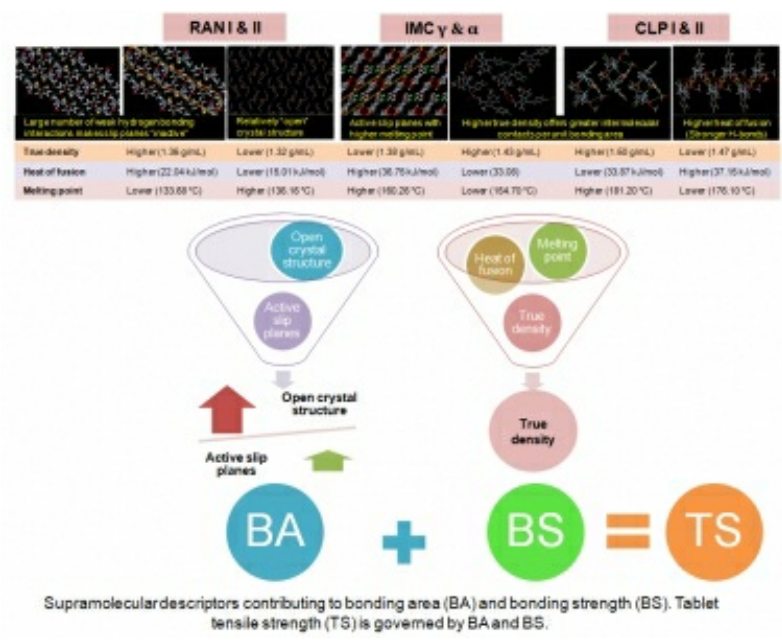

Keywords: crystal structure, mechanical properties, tableting 